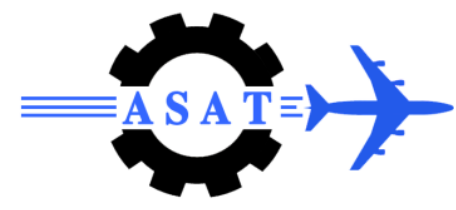

\title{
Error Models for MEMS Based IMU
}

\author{
M.K. El Bahnasawy ${ }^{*}$, E. Abdelkawy ${ }^{\dagger}$, S.A. Shedied ${ }^{*}$
}

\begin{abstract}
This paper introduces a comparison study of different error models used in strapdown inertial navigation system (SINS) to enhance the overall positioning accuracy. These models are used to represent the stochastic errors included in the MEMS sensors used in the IMU. First-order Gauss-Markov (GM) model, Random walk (RW) model and autoregressive (AR) process model are used to describe the stochastic nature of these errors and their effect on positioning accuracy.
\end{abstract}

Keywords: MEMS, Micro Electromechanical Systems, INS, Inertial Navigation Systems

\section{Introduction}

The last two decades have shown an increasing demand for low-cost and miniature inertial sensors in many navigation applications. However MEMS-based inertial sensors efficiently meet this demand, due to their lightweight and fabrication process. MEMS sensors still have some performance limitations, which consequently affect their obtainable accuracy and sensitivity. One of the main problems of MEMS sensors is that the remaining uncertainties of the sensor errors which are very large and sensitive to surrounding environmental changes [1].

Integration between MEMS-based inertial sensors and another aiding system, like GPS, has become one of the most important and attractive low-cost solutions to navigation applications. However, the most important challenge when working with MEMS INS/GPS is to develop an integration algorithm (estimators) that can deal with the large instrument errors and the corrupted GPS data in signal-degraded environment. The INS/GPS integration is commonly performed using Kalman filter (KF) estimator. When a GPS outage occurs, KF operates in prediction mode, correcting the INS information based on the system error model. KF has widely been used for data fusion and is considered as the most famous tool for INS/GPS integration. The most commonly used integration scheme in many literatures is loosely (used in this application) and tightly coupled integration schemes.

In loosely coupled integration algorithm, the INS and the GPS receiver operate as independent systems and process data in parallel. INS raw measurements (acceleration and angular velocity) are processed in the INS mechanization to derive INS attitude, velocity and position. GPS raw observations (code, Doppler and phase) are to derive GPS velocity and position. The INS Kalman filter utilizes the differences between the INS and GPS velocities and positions as the measurements and the INS error equations as the system model. When

\footnotetext{
*Egyptian Armed Forces, Egypt.

† Egyptian Armed Forces, Egypt.

Egyptian Armed Forces, Egypt, samyshedied@msn.com
} 
GPS is available, the INS Kalman filter estimates all observable INS sensor and navigation errors to compensate system outputs. When GPS is unavailable, INS sensor and navigation errors will be predicted based on the system model [2].

In the tightly coupled integration both INS data and GPS raw measurements are processed together. Similarly to the loosely coupled integration algorithm, the INS navigation states are first derived from the INS raw measurements based on the INS mechanization. Then, in the INS/GPS Kalman filter the INS sensor and navigation errors as well as GPS range and range rate errors are estimated using the pseudo ranges and delta ranges calculated by the INS and measured by a GPS receiver as the system measurements. The estimated INS errors will be applied to correct the INS navigation states.

According to whether the estimated sensor errors are fed back to correct the measurements, both loosely and tightly coupled integration algorithm can be implemented with an open loop or closed loop. The closed-loop implementation, generally enhances the navigation performance because the previous estimation results are used to minimize the error approximation due to system model linearization, are mostly applied in INS/GPS integration systems.

\section{Kalman Filtering}

The Kalman Filter (KF) is an efficient stochastic estimator for a large number of problems, especially in the field of navigation. It is a recursive predictive estimator that is based on the use of state space technique and recursive algorithms. KF consists of two main steps: prediction and correction. It depends on minimization of the mean square error between the prediction of parameters from a previous time instant and external observations at a present time instant.

According to linear system theory, a state space model can represent the dynamics of a linear system, where a set of first order differential equations express the deviation from a reference trajectory as:

$$
\begin{aligned}
& \dot{\chi}=F \chi+w \\
& z=\mathrm{H} \chi+v
\end{aligned}
$$

For a discrete system, the state transition matrix $\Phi$ can be approximated by a Taylor series expansion over a short time interval $\Delta \mathrm{t}$, after the first two terms, results in:

$$
\Phi \approx I+F \Delta t
$$

Kalman filtering process can be summarized in two-step, the first step is prediction by the system model, i.e.:

$$
\begin{array}{ll}
\text { State estimate } & \hat{\chi}_{\mathrm{k}+1}^{-}=\Phi_{\mathrm{k}} \hat{\chi}_{\mathrm{k}} \\
\text { Error covariance estimate } & \mathrm{P}_{\mathrm{k}+1}^{-}=\Phi_{\mathrm{k}} \mathrm{P}_{\mathrm{k}} \Phi_{\mathrm{k}}^{\mathrm{T}}+\mathrm{Q}_{\mathrm{k}}
\end{array}
$$

and the second step is the measurement update of the system model. The elements of the update process are as follow: 


$$
\begin{array}{lll}
\text { Kalman gain matrix } & \mathrm{K}_{\mathrm{k}}=\mathrm{P}_{\mathrm{k}}^{-} \mathbf{H}_{\mathrm{k}}^{\mathrm{T}}\left[\mathrm{H}_{\mathrm{k}} \mathbf{P}_{\mathrm{k}}^{-} \mathbf{H}_{\mathrm{k}}^{\mathrm{T}}+\mathrm{R}_{\mathrm{k}}\right]^{-1} \\
\text { Error covariance update } & \mathrm{P}_{\mathrm{k}}=\left[\mathrm{I}-\mathrm{K}_{\mathrm{k}} \mathrm{H}_{\mathrm{k}}\right] \mathrm{P}_{\mathrm{k}}^{-} \\
\text {State update } & \hat{\chi}_{\mathrm{k}}=\hat{\chi}_{\mathrm{k}}^{-}+\mathrm{K}_{\mathrm{k}}\left[\mathrm{Z}_{\mathrm{k}}-\mathrm{H}_{\mathrm{k}} \hat{\chi}_{\mathrm{k}}^{-}\right]
\end{array}
$$

In this paper, we will derive the error model for the MEMS based IMU with different techniques to be involved in the KF algorithm and the effect on the performance of the final navigation track of the vehicle.

\section{Error Models for MEMS IMU}

Errors in INS result from the following two sources: 1) the sensor itself and 2) the numerical integration process in the INS mechanization. MEMS inertial sensors have both deterministic and random errors. The deterministic errors can be obtained using calibration procedures and then removed from the raw measurements, but the random errors must be modeled stochastically to reduce their effect on the positioning accuracy [1].

The MEMS sensor measurement equation models can be considered as:

$$
\begin{gathered}
\tilde{f}_{-}^{b}=f_{-}^{b}+\underline{b}_{a}+\operatorname{diag}\left(\underline{f}_{-}^{b}\right) S_{a}+w_{a} \\
\underline{\tilde{\omega}}_{i b}^{b}=\underline{\omega}_{i b}^{b}+\underline{b}_{g}+\operatorname{diag}\left(\underline{\omega}_{i b}^{b}\right) S_{g}+w_{a}
\end{gathered}
$$

where $\tilde{f_{-}^{b}}$ is the measured accelerometer is vector and $f_{-}^{b}$ is the true accelerometer vector. $\tilde{\omega}_{i b}^{b}$ is the measured gyroscope vector and $\underline{\omega}_{i b}^{b}$ is the true gyroscope vector. $\underline{b}_{a}$ and $\underline{b}_{g}$ are the biases of accelerometer and gyroscope respectively. $S_{a}$ and $S_{g}$ are scale factors of accelerometer and gyroscope respectively[2].Finally, the accelerometer and gyroscope additive white Gaussian noise are represented by $w_{\mathrm{a}}$ and $w_{\mathrm{g}}$ respectively.

In this section, 3 models for MEMS sensor errors will be reviewed as follows:

\subsection{Random Walk}

In this case the sensor error is assumed to be a white noise as shown in Fig. (1), the continuous-time state equation for random walk is given by [6]:

$$
\dot{x}=w
$$

The corresponding discrete-time process state equation is:

$$
x_{k+1}=x_{k}+w_{k}
$$

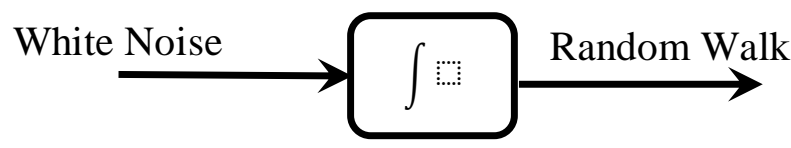

Figure (1) White noise and random walk 


\subsection{Gauss-Markov Process}

In this method, the sensor error is modelled as a stationary Gaussian that has an exponentially decaying autocorrelation is called first-order Gaussian Markov process. For a process of correlation time $\mathrm{T}$ and root mean square value (data standard deviation) $\sigma$, the continuoustime model is described by:

$$
\dot{x}=\frac{-1}{\mathrm{~T}} x+w
$$

The corresponding discrete-time process model is given by:

$$
x_{k+1}=e^{-\Delta t_{k+1 / T}} x_{k}+w_{k}
$$

Therefore, Gaussian Markov process is very important as it fits large number of physical processes and it has a relatively simple mathematical description. Autocorrelation function (ACF) and closed loop system representing first order GM process is shown in Fig. (2-a) and (2-b) respectively.

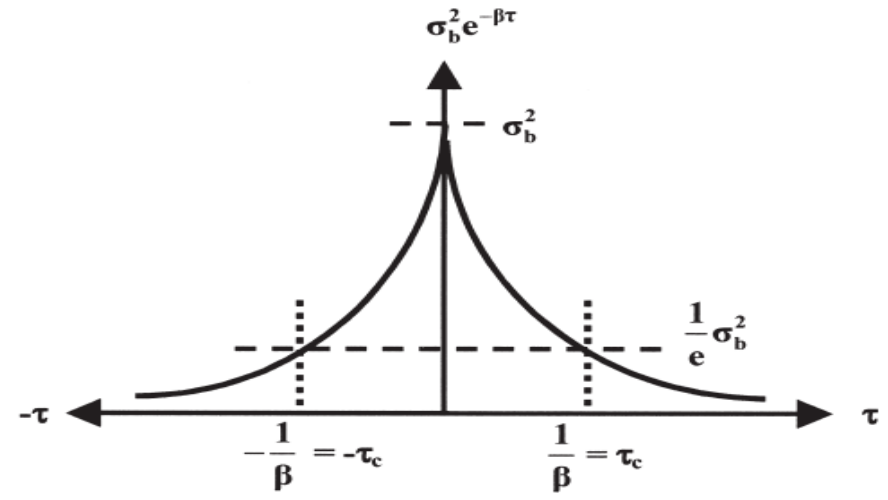

a. Autocorrelation function (ACF)

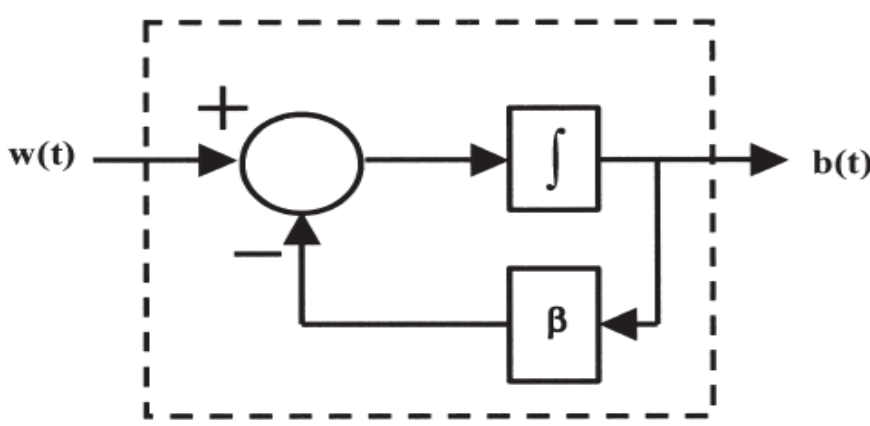

b. Closed loop system

Fig. (2) $1^{\text {st }}$ order GM process

\subsection{Autoregressive Process}

The term "autoregressive" comes from the fact that each signal sample is regressed on (or predicted from) the previous values of itself. The AR transfer function relationship can be obtained in time domain as [1]:

$$
y(n)=-\sum_{k=1}^{p} a_{k} y(n-k)+b_{0} w(n)
$$

This is written as 


$$
y(n)=-a_{1} y(n-1)+-a_{2} y(n-2)-\ldots-a_{p} y(n-1)
$$

In time and frequency domain, the AR transfer function relationship can be shown in the Fig. (3):

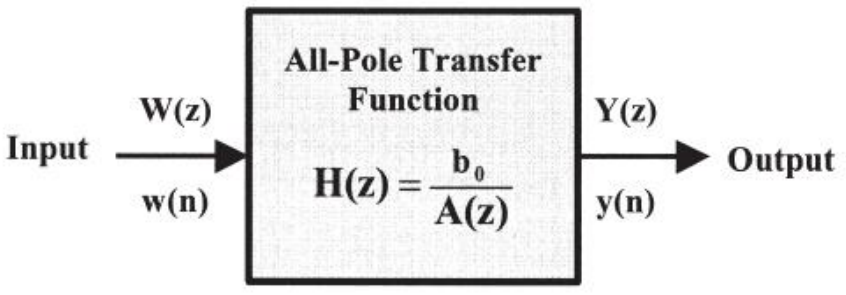

\section{Figure (3) Transfer function relationship} of autoregressive function

Several methods have been reported for estimating the parameter values by fitting an AR model to the input data. Three main methods are usually used: the Yule-Walker method, the covariance method, and Burg's method. In principle, all of these estimation techniques should lead to approximately the same parameter values if fairly large data samples are used.

The Yule-Walker method has inadequate performance in the case of short data records because of the data windowing applied by its algorithm. Moreover, that method may introduce a large bias in the AR estimated coefficients since it does not guarantee a stable solution of the model. The covariance method is similar to the Yule-Walker method in that it minimizes the prediction error in the least-squares sense, but it does not consider any windowing of the data.

However, unstable AR models may be obtained if the covariance method is used. Burg's method was introduced to overcome most of the drawbacks of the other AR modeling techniques by providing both stable models and high resolution (i.e., more accurate estimates) for short data records [8].

\section{Experimental Work}

In this paper, SINS error modeling will study only parameters of 2-D land vehicle navigation (latitude " $\varphi$ ", longitude " $\lambda$ ", north velocity " $\mathrm{V}_{\mathrm{n}}$ ", east velocity " $\mathrm{V}_{e}$ " and yaw (azimuth) angle " $\psi$ " of the state vector. X-accelerometer, Y-accelerometer and Z-Gyro are IMU errors that are modeled stochastically and included in the SINS error model.

First, the IMU data is collected in stationary for long time (approximately 8 hours) and studied to get the required parameters and coefficients (as: correlation time, data standard deviation and variance, AR coefficients) used in stochastic error modeling in Kalman filter equations.

\section{1 $1^{\text {st }}$ order GM Model}

The KF state vector consists of 8 states ( 5 states of the INS error model and additional 3 states of sensor error model) as shown in Eq. (17):

$$
x=\left[\begin{array}{llllllll}
\delta \phi & \delta \lambda & \delta \mathrm{V}_{n} & \delta \mathrm{V}_{e} & \delta \mathrm{y} & \delta \mathrm{f}_{\mathrm{x}} & \delta \mathrm{f}_{\mathrm{y}} & \delta \omega_{\mathrm{z}}
\end{array}\right]^{\mathrm{T}}
$$

The state transition matrix $(\Phi)$ which is the dynamic part in the system can be calculated in the KF error state model as shown in Eq. (18): 


$$
\boldsymbol{\Phi}=\left[\begin{array}{cccccccc}
1 & 0 & \Delta t & 0 & 0 & 0 & 0 & 0 \\
0 & 1 & 0 & \Delta t & 0 & 0 & 0 & 0 \\
0 & 0 & 1 & 0 & -a_{x} \Delta t & \cos \psi \Delta t & -\sin \psi \Delta t & 0 \\
0 & 0 & 0 & 1 & -a_{y} \Delta t & \sin \psi \Delta t & \cos \psi \Delta t & 0 \\
0 & 0 & 0 & 0 & 1 & 0 & 0 & \Delta t \\
0 & 0 & 0 & 0 & 0 & 1-\beta_{a_{x}} & 0 & 0 \\
0 & 0 & 0 & 0 & 0 & 0 & 1-\beta_{a_{y}} & 0 \\
0 & 0 & 0 & 0 & 0 & 0 & 0 & 1-\beta_{\omega_{z}}
\end{array}\right]
$$

$\beta$ is the reciprocal of the correlation time $(\tau)$ which is derived from static sensor data autocorrelation function.

The KF measurement vector $(\mathrm{Z})$ can be derived using the difference between the GPS-derived position estimates, and the INS mechanization-derived position to obtain the error estimates represented as shown in Eq. (19):

$$
\mathrm{Z}_{\mathrm{k}}=\left[\left(\Phi_{\mathrm{INS}}-\Phi_{\mathrm{GPS}}\right)\left(\lambda_{\mathrm{INS}}-\lambda_{\mathrm{GPS}}\right)\right]^{\mathrm{T}}
$$

The measurement design matrix $(\mathrm{H})$ for GM model is shown in Eq.(20):

$$
\mathrm{H}_{2 \times 8}=\left[\begin{array}{ll}
\mathrm{I}_{2 \times 2} & \mathrm{O}_{2 \times 6}
\end{array}\right]
$$

\subsection{Random Walk Model}

This KF model is the same as GM model but with small differences in the state transition matrix elements $(\Phi(6,6), \Phi(7,7)$ and $\Phi(8,8))$ are equal "one".

\subsection{Autoregressive Model}

First-order GM model is usually proposed for navigational and tactical grade inertial sensors. For MEMS inertial sensors, the assumption that the sensor random errors follow the stochastic nature of a first-order GM model is not always valid. Using a stationary run, the autocorrelation sequences of the MEMS grade Crossbow IMU300CC module were calculated as shown in Fig. 5.

By comparing these ACFs, it can be shown that the random errors associated with these MEMS inertial sensors may be more adequate to be modeled with AR error modeling technique than a first-order GM process.

Two main issues in AR stochastic error modeling should be determined: 1) parameter estimation method and 2) AR order. Parameter estimation method could be, as mentioned before, Yule-walker, Covariance or Burg's method which is used here because it gives better performance [8]. Stationary sensor measurements are used also for selecting the order AR model. Fig. 6 shows that $2^{\text {nd }}$ order AR error model (in our case) will be suitable for modeling inertial sensor errors. It should be noted the increasing order of AR error model will increase states of K.F. which may results in filter instabilities. 

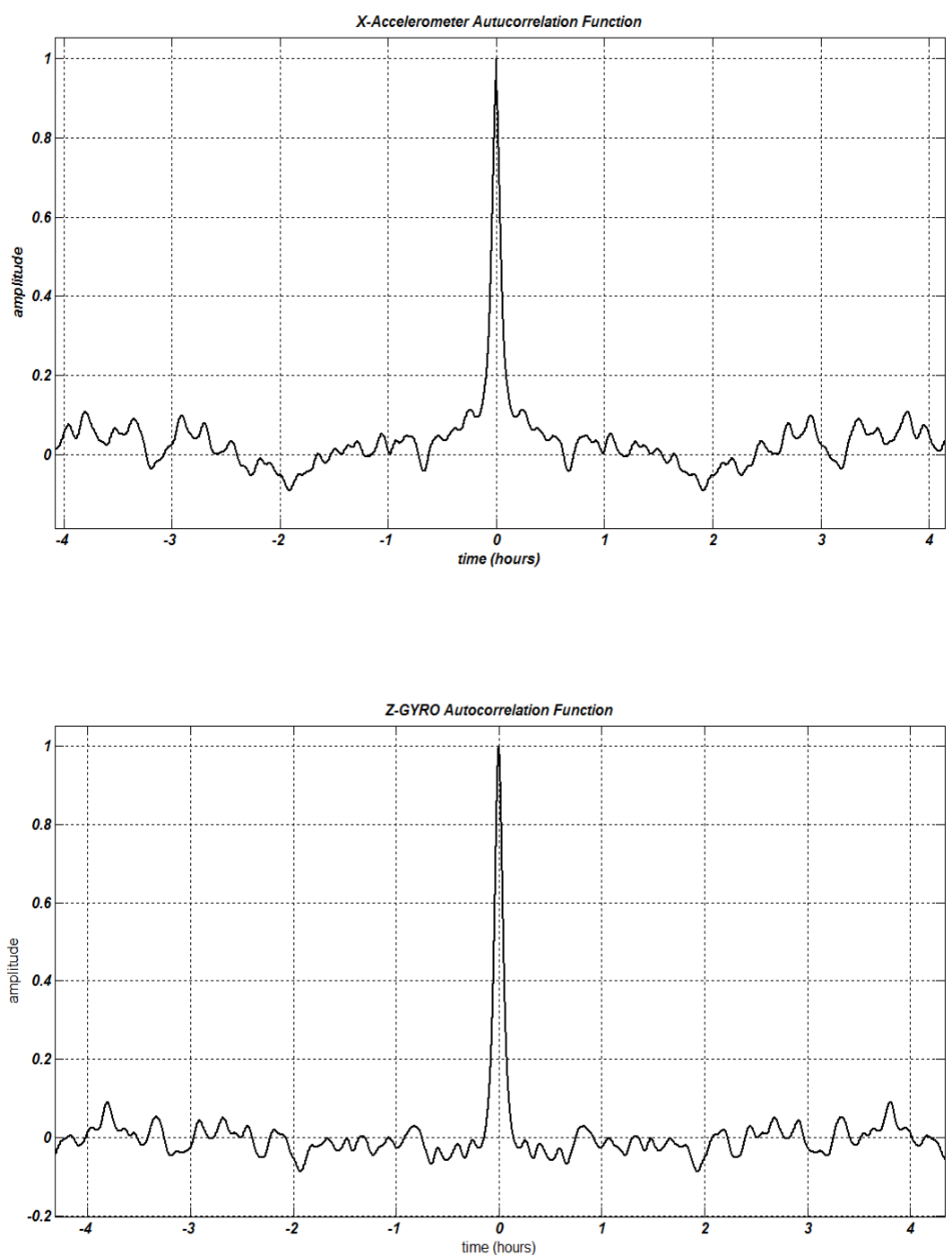

Figure (5): Autocorrelation sequences of $X$-accelerometer (above) and Z-Gyro (down)

The KF state vector consists of 8 states ( 5 states of the INS error model and additional 2 states for modeling each one of the 3 sensors) as shown in Eq. (21):

$$
x=\left[\begin{array}{lllllll}
\delta \phi & \delta \lambda & \delta \mathrm{V}_{n} & \delta \mathrm{V}_{e} \delta \mathrm{y} \delta \mathrm{f}_{\mathrm{x} 1} \delta \mathrm{f}_{\mathrm{x} 2} \delta \mathrm{f}_{\mathrm{y} 1} \delta \mathrm{f}_{\mathrm{y} 2} \delta \omega_{\mathrm{z} 1} \delta \omega_{\mathrm{z} 2}
\end{array}\right]^{\mathrm{T}}
$$

The state transition matrix $(\Phi)$ which is the dynamic part in the system can be calculated as shown in Eq. (22). 

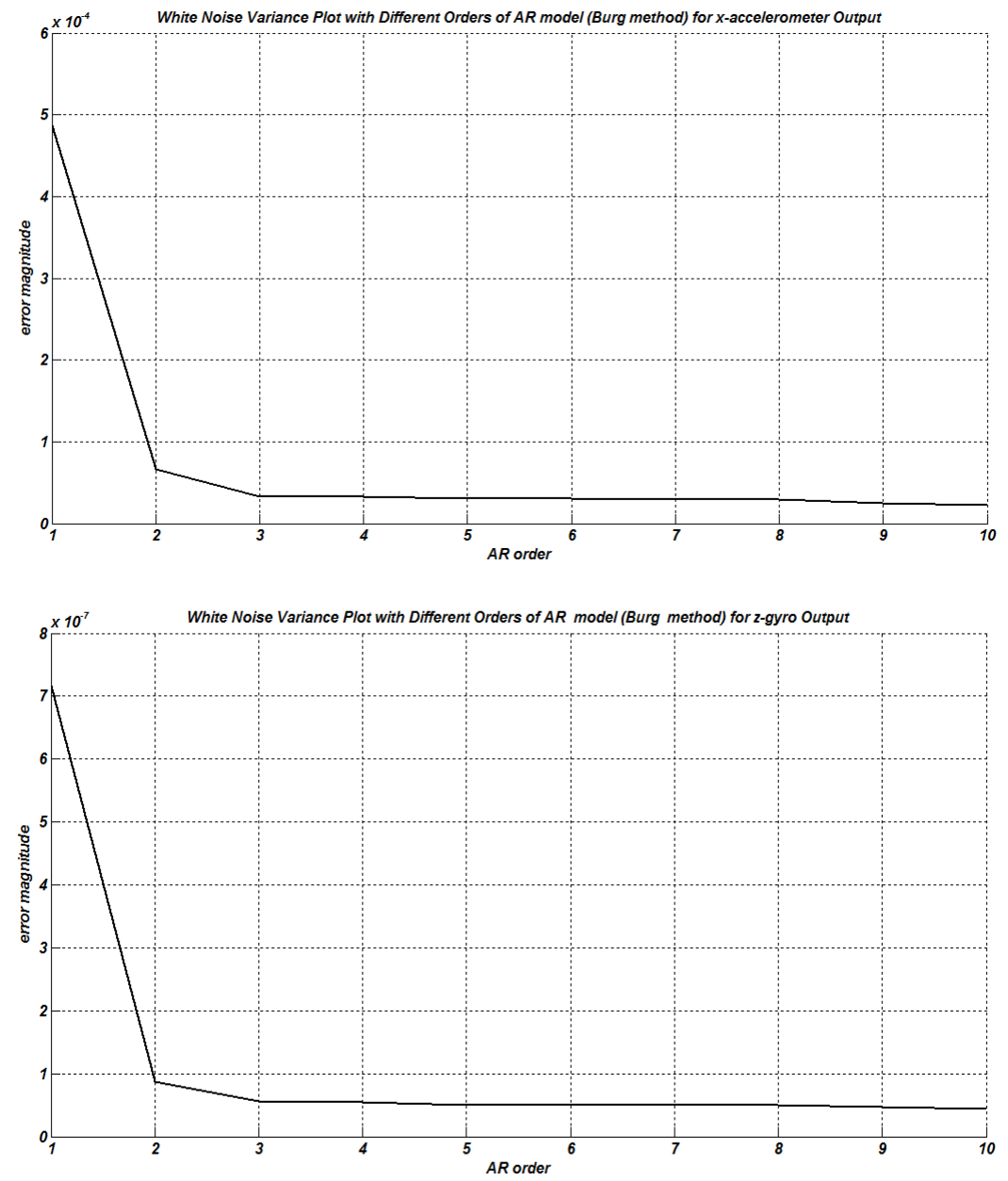

Figure (6) AR Model Prediction RMSE of X-accelerometer (above) and Z-Gyro (down)

$\boldsymbol{\Phi}=\left[\begin{array}{ccccccccccc}1 & 0 & \Delta t & 0 & 0 & 0 & 0 & 0 & 0 & 0 & 0 \\ 0 & 1 & 0 & \Delta t & 0 & 0 & 0 & 0 & 0 & 0 & 0 \\ 0 & 0 & 1 & 0 & -a_{x} \Delta t & \cos \psi \Delta t & 0 & -\sin \psi \Delta t & 0 & 0 & 0 \\ 0 & 0 & 0 & 1 & -a_{y} \Delta t & \sin \psi \Delta t & 0 & \cos \psi \Delta t & 0 & 0 & 0 \\ 0 & 0 & 0 & 0 & 1 & 0 & 0 & 0 & 0 & \Delta t & 0 \\ 0 & 0 & 0 & 0 & 0 & -a_{1}^{a_{x}} & -a_{2}^{a_{x}} & 0 & 0 & 0 & 0 \\ 0 & 0 & 0 & 0 & 0 & 1 & 0 & 0 & 0 & 0 & 0 \\ 0 & 0 & 0 & 0 & 0 & 0 & 0 & -a_{1}^{a_{y}} & -a_{2}^{a_{y}} & 0 & 0 \\ 0 & 0 & 0 & 0 & 0 & 0 & 0 & 0 & 0 & 0 & 0 \\ 0 & 0 & 0 & 0 & 0 & 0 & 0 & 0 & 0 & -a_{1}^{\omega_{z}} & -a_{2}^{\omega_{z}} \\ 0 & 0 & 0 & 0 & 0 & 0 & 0 & 0 & 0 & 1 & 0\end{array}\right]$

$a_{1}^{a_{x}}, a_{2}^{a_{x}}, a_{1}^{a_{y}}, a_{2}^{a_{y}}, a_{1}^{\omega_{z}}, a_{2}^{\omega_{z}}$ are the coefficients of $2^{\text {nd }}$ order AR error model for ax , ay and $\omega_{\mathrm{z}}$ respectively.

The measurement design matrix $(\mathrm{H})$ for $\mathrm{AR}$ model is shown in Eq. (23):

$$
\mathrm{H}_{2 \times 11}=\left[\begin{array}{ll}
\mathrm{I}_{2 \times 2} & 0_{2 \times 9}
\end{array}\right]
$$




\subsection{Results Discussion}

The trajectories of the integrated system using $2^{\text {nd }}$ order AR model, $1^{\text {st }}$ order GM model and RW model were generated with the $1-\mathrm{sec}$ update then it is compared with the reference Precise Point Positioning (PPP) trajectory. After that, the system trajectories were generated with 3-sec and 6-sec update intervals that are illustrated in from Fig. 7 to Fig. 9.

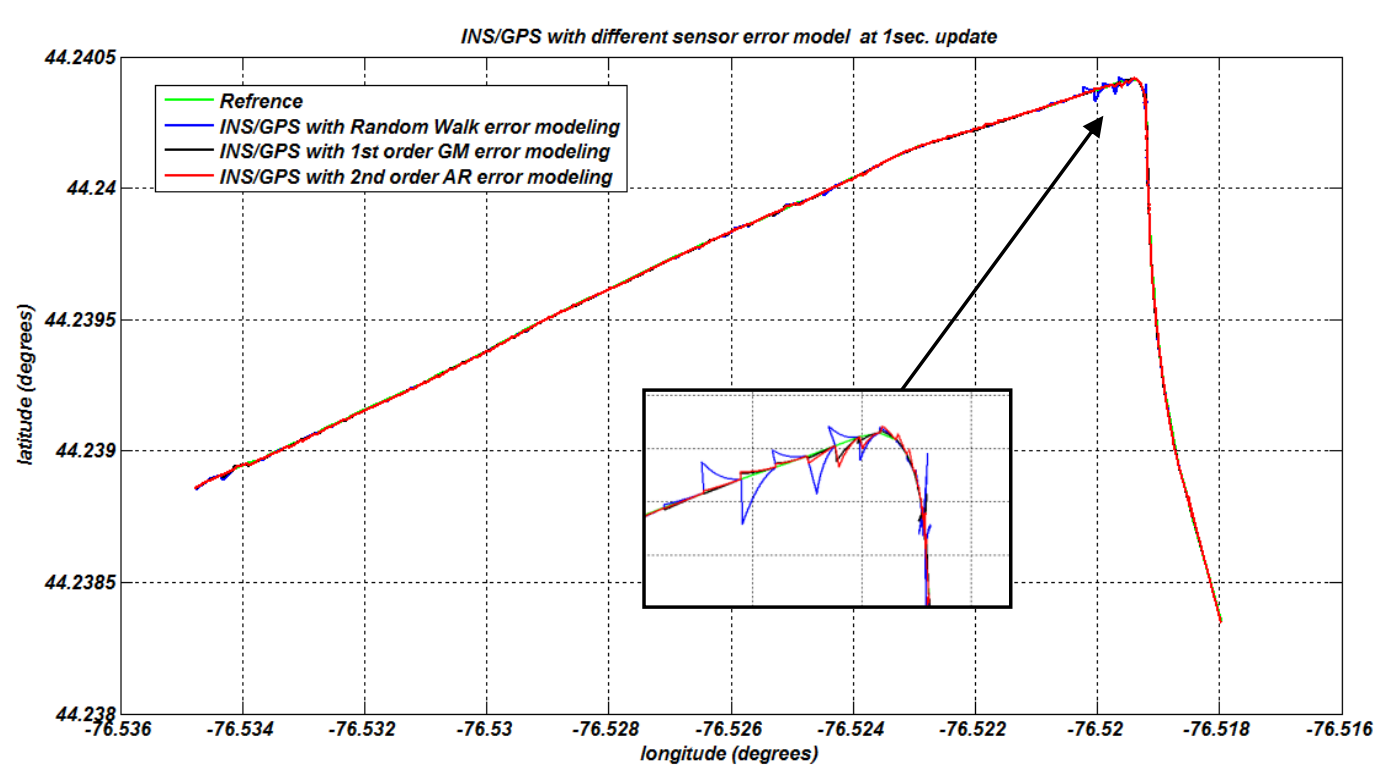

Figure (7) : RW, 1st order GM and 2nd order AR integration models with 1sec. update

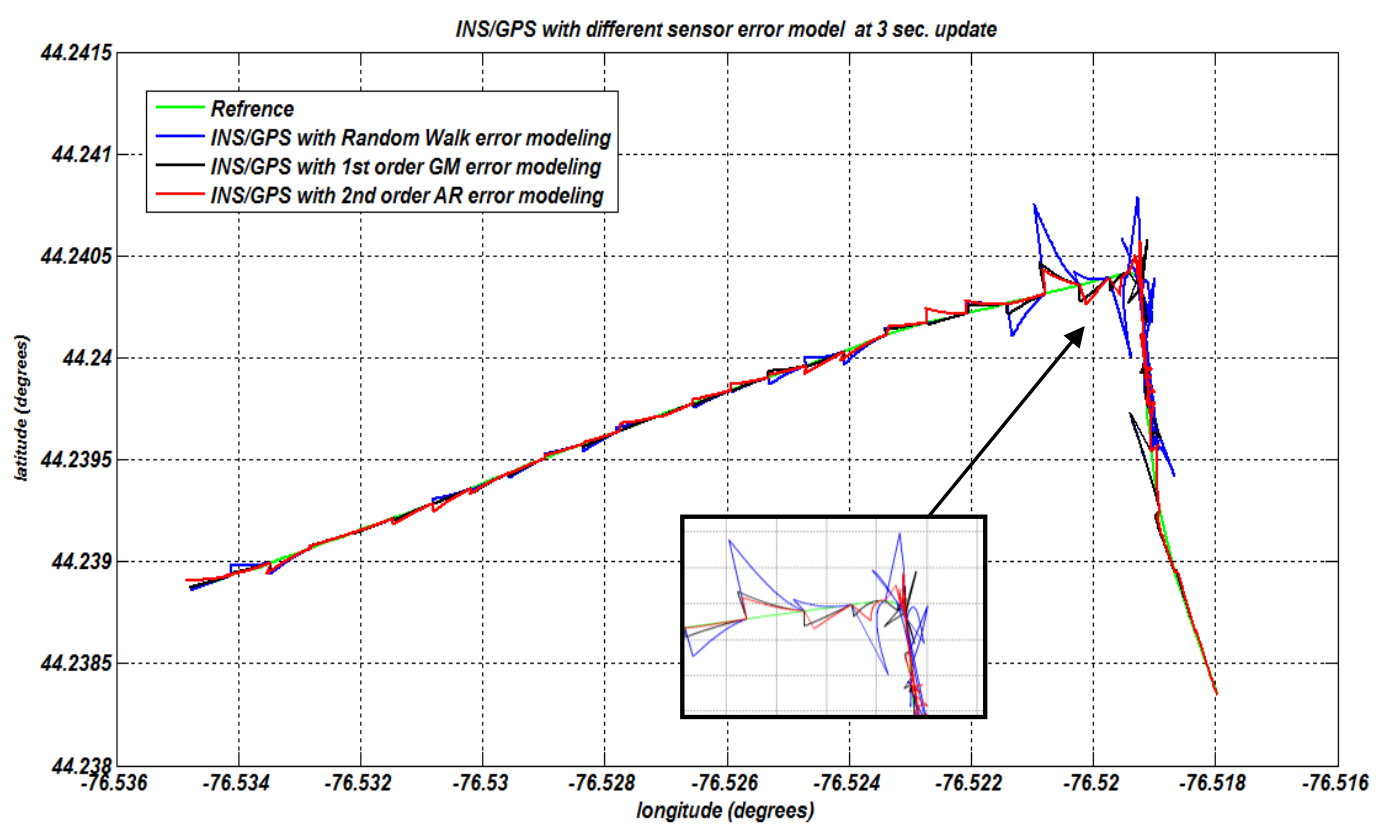

Figure (8) RW, 1st order GM and 2nd order AR integration models with 3sec. update 


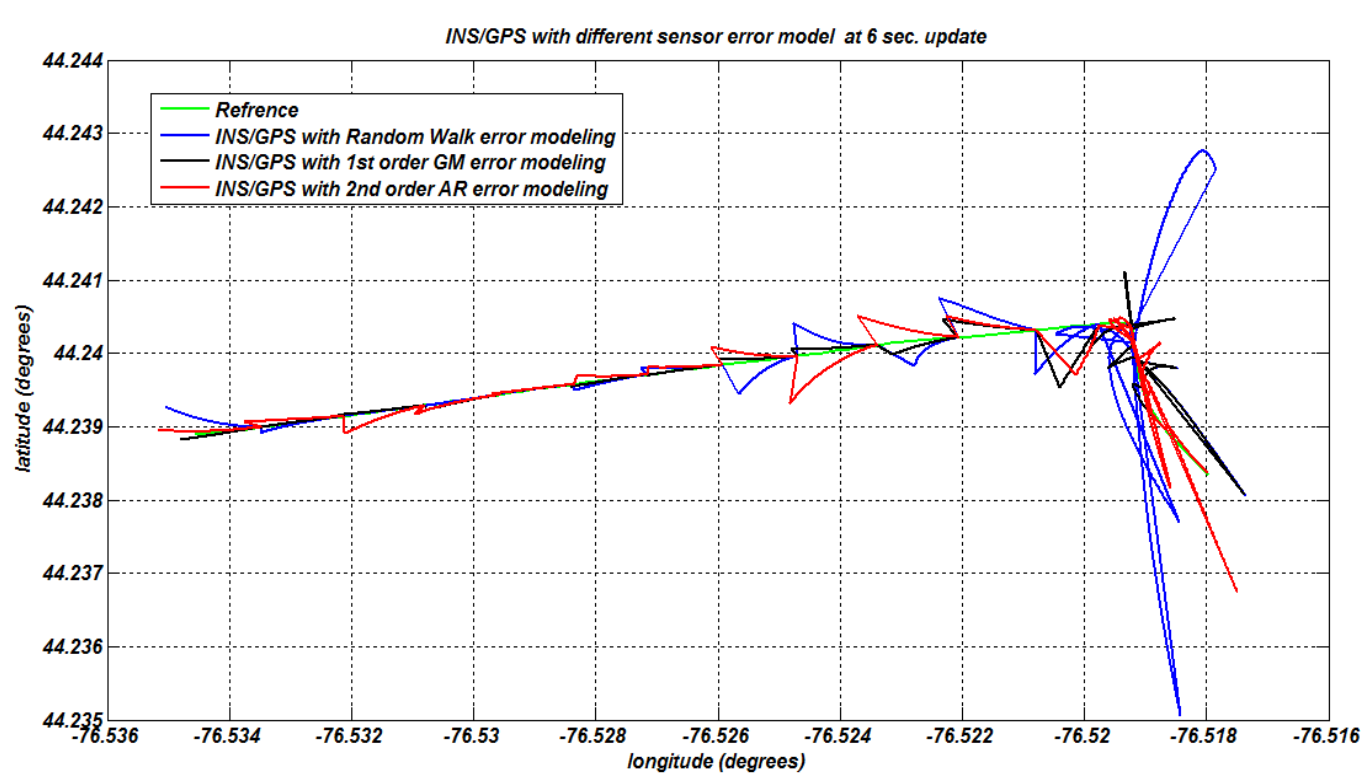

Figure (9) RW, 1st order GM and 2nd order AR integration models with 6sec. update

\subsection{Conclusion}

From the above figures we can conclude that $2^{\text {nd }}$ order AR model gives better solution than $1^{\text {st }}$ order GM model and RW model especially at maneuvering points. It is shown that increasing update intervals deteriorates the integration performance and it clearly appears when increasing update intervals to 6 seconds. So, it is advisable to use this INS/GPS integration scheme with $2^{\text {nd }}$ order AR model for the above used sensors until 5 seconds (as maximum) to get results with acceptable error levels.

\section{References}

[1] A.Noureldin, Tashfeen B. Karamat and Ahmed El-Shafie, Performance Enhancement of MEMS-Based INS/GPS Integration for Low-Cost Navigation Applications, IEEE TRANSACTIONS ON VEHICULAR TECHNOLOGY, VOL. 58, NO. 3, MARCH 2009.

[2] Joshy Madathiparambil Jose, Performance Enhancement of Extended and Unscented Kalman Filter Implementation in GPS-INS Integration, MSc, Prague 2009

[3] S.Nassar, A.Noureldin and N.El Shemy, Modeling Inertial Sensor Errors Using Autoregressive (AR) Models, 2008.

[4] Minha Park and Yang Gao, Error and Performance Analysis of MEMS -based Inertial Sensors with a Low-cost GPS Receiver, Sensors 2008.

[5] Saurabh Godha, Performance Evaluation of Low Cost MEMS-Based IMU Integrated With GPS for Land Vehicle Navigation Application, PhD, February 2006

[6] Eun-Hwan Shin, Estimation Techniques for Low Cost Inertial Navigation, $\mathrm{PhD}$, May2005, CALGARY, ALBERTA, CANADA

[7] S. Godha and M. E. Cannon, Integration of DGPS with a Low Cost MEMS - Based Inertial Measurement Unit (IMU) for Land Vehicle Navigation Application, ION GPS 05, September13-16, 2005, Long Beach, CA.

[8] Sameh Nassar, Improving the Inertial Navigation System (INS) Error Model for INS and INS/DGPS Applications, PhD, November 2003, CALGARY, ALBERTA, CANADA. 